\section{On the tiles}

Nature 454, 501-504 (2008)

Quasicrystals are a curiosity: they are aperiodic, but still have long-range order. Thin films deposited on quasicrystalline surfaces may also take on quasicrystalline properties and could be ripe for exploitation, if their growth mechanism were fully understood.

Jules Mikhael and colleagues have performed an elegant study of the deposition of a colloidal monolayer on a quasicrystalline substrate. Their substrate, in fact, is a potential, created from the interference of five laser beams. At zero or low potential, the charged polystyrene spheres of the colloid (suspended in water) settle into a crystal that can be described by a lattice of triangular tiles. At higher potential, and when the ionic strength of the colloidal suspension is such that interactions between the colloid and the

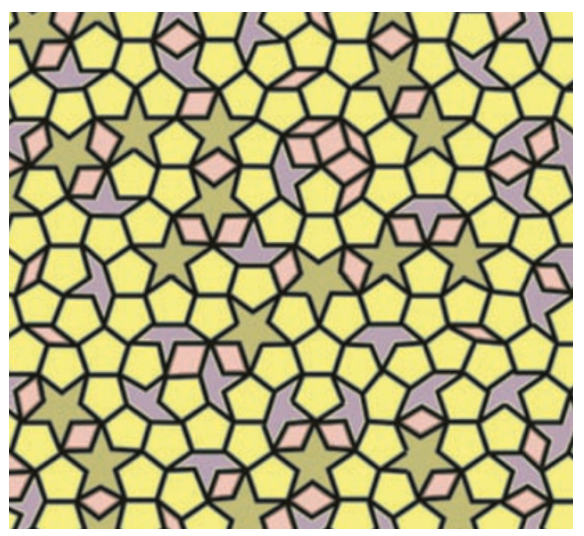

substrate become dominant, a quasicrystal results, its structure matched by a pattern of rhombuses, pentagons, crowns and stars.

Between these two phases, at intermediate potential, a different type of quasicrystal develops - matched by a pattern of squares and triangles that is similar to one of the so-called archimedean tilings, introduced by Johannes Kepler in 1619, and currently of interest in the design of photonic crystals.

\section{New-age resonator}

\section{Nature Nanotech.}

\section{doi:10.1038/nnano.2008.200 (2008)}

One of the most common uses of mechanical resonators is as simple but sensitive mass detectors. This takes advantage of the fact that even small changes to the mass of resonator such as by the adsorption of material from the surrounding environment on its surface can shift the frequency at which it resonates.

Kenneth Jensen and colleagues have pushed this approach to the atomic limit, with a resonant mass sensor built using a suspended carbon nanotube. Owing to their high mechanical stiffness, carbon nanotubes resonate at much higher frequencies than nanoscale resonators built by conventional lithographic techniques. And their light weight makes their resonant behaviour more susceptible to changes in their mass: the device can reach a detection sensitivity of just 0.13 zeptogram $\mathrm{Hz}^{-1 / 2}$ - equivalent to just 0.4 gold atoms per $\mathrm{Hz}^{1 / 2}$.

\section{Fast and cheap}

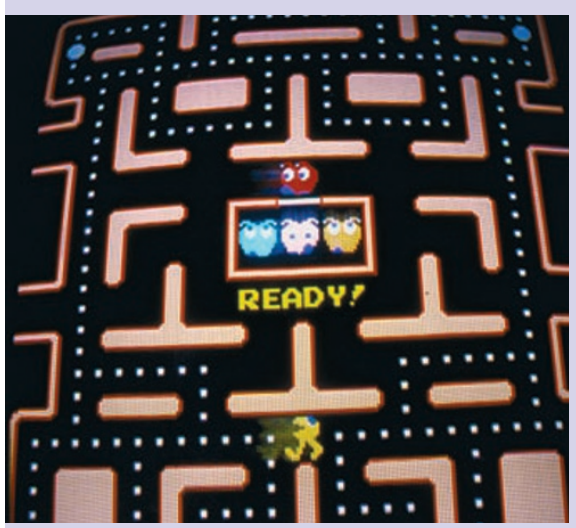

J. Comput. Phys.

doi:10.1016/j.jcp.2008.05.023 (2008)

The plots of video games might not be to all tastes. But, at least in terms of the graphics, each generation of game brings fresh wonder as the worlds into which the players are plunged become increasingly sophisticated and realistic. The graphics processing units (GPUs) that calculate these fantasies are becoming ever more powerful, and cheaper: Nail Gumerov and Ramani Duraiswami suggest that they could be of good service to scientists, outside of playtime.

Gumerov and Duraiswami have mapped the so-called fast multipole method onto a GPU architecture. Fast multipole methods are algorithms that enable the efficient calculation of long-range forces in $\mathrm{N}$-body problems, and are used in a wide range of fields, from molecular and fluid dynamics to astrophysics. After optimizing all components of the algorithm, the authors achieved calculation speeds that were 30 to 60 times faster, compared with similar implementations on a general-purpose central processing unit, or CPU. It seems that GPUs - their development constantly driven by the mass market for games might indeed prove a useful possibility for scientific computing.
At such extreme sensitivity, the authors observe fluctuations in the device's response arising from the random absorption of discrete atoms, analogous to the shot noise seen in sensitive electronic devices.

\section{Spin on relativity}

Science 321, 104-107 (2008)

The general theory of relativity has passed many tests in both the weak- and the strongfield regimes of gravity. However, relativistic contributions due to gravitation-mediated spin-orbit and spin-spin coupling have been harder to probe and verify. Using highprecision timing data from a double-pulsar system, Rene P. Breton and colleagues have measured relativistic spin precession and use it to test general relativity, and to constrain alternative theories of gravity.

In the binary system PSR J0737-3039A/B, both neutron stars are radio pulsars and have much higher mean orbital velocities than other binary pulsars. The spin-orbit coupling - the interaction between the spin of a compact body in a binary system with the orbital angular momentum - gives rise to a relativistic spin precession. From data acquired between 2003 and 2007, Breton et al. measured the relativistic spin precession rate to within a $13 \%$ uncertainty of what is predicted by general relativity - suggesting that the theory is valid in the strong-field regime of gravity, even for spinning bodies.

\section{A tunable mix}

Phys. Rev. Lett. (in the press); preprint at http://arxiv.org/abs/0802.2591 (2008)

The spatial separation of immiscible fluids is a common phenomenon, but Scott Papp and colleagues have found a similar effect in a Bose-Einstein condensate of two atomic species - where such behaviour is a surprise.

Papp et al. created a mixture of rubidium85 and rubidium-87 atoms, expecting, from theory, that the density patterns for the two species should reflect the symmetry of the external trapping potential. Instead, however, images of the condensate showed spatially separated domains in which only one of the isotopes was present. Depending on the relative number of atoms of each species, as many as three such 'cloudlets' appeared, providing clear evidence that the two Bosecondensed species are immiscible.

The degree of separation could also be controlled. By tuning the interparticle interactions - using an applied magnetic field, which changes the scattering length the behaviour of the condensate could be changed back and forth between miscible and immiscible. 